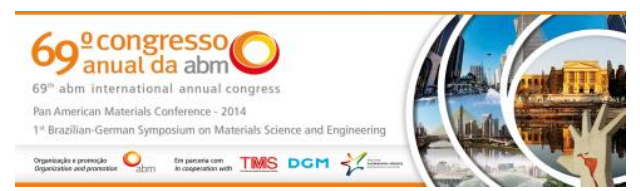

Tema: Materiais Cerâmicos, Compósitos e Poliméricos

\title{
CARACTERIZAÇÃO EXPERIMENTAL DO PROCESSO DE DELAMINAÇÃO EM MATERIAL COMPÓSITO DE FIBRA DE CARBONO E RESINA EPÓXI*
}

\author{
Amanda Coutinho Campanatti ${ }^{1}$
} Gigliola Salerno ${ }^{2}$

\section{Resumo}

O objetivo desse trabalho é caracterizar o processo de delaminação, tanto em Modo I como em Modo II, de abertura de trinca. A densidade e porcentagem de fibra do compósito carbono-epóxi foram calculadas para caracterizar o compósito. O inserto, para simular o início da trinca, foi feito no processo de fabricação dos compósitos a fim de manter sua integridade. Pelo mesmo motivo o corte foi feito a jato d'água. Para caracterização da delaminação foram feitos ensaios de DCB (Double-cantilever Beam) e ENF (End-notched Flexure), segundo as normas ASTM D5528 e ASTM D790-10, sendo ambos realizados na máquina universal de ensaio MTS, capacidade de $250 \mathrm{kN}$. A fractografia em Microscopia Eletrônica de Varredura (MEV) foi feita a fim de identificar os mecanismos de dano durante a propagação da trinca. Foi possível concluir a fabricação do compósito, bem como do inserto, é viável. Porém, houve aparecimento de bolhas, por causa da baixa pressão da bomba a vácuo. 0 corte a jato d'água manteve a integridade do compósito. A rigidez alta do compósito pode ser comprovada por meio das propriedades mecânicas obtidas pelo ensaio de tração. Os valores de $G_{ı c}$ e G॥c estão dentro do esperado pela literatura, sendo G॥c maior que Gı por causa da presença de um capo de compressão no ensaio em modo II, enquanto que em modo I é abertura pura por tração.

Palavras-chave: Delaminação; Compósito; Fibra de carbono.

\section{CARBON EPOXY COMPOSITE MATERIAL EXPERIMENTAL DELAMINATION}

\begin{abstract}
The main purpose of this study is the delamination characterization in Modes I and II. Density and fibre quantity were identified in order to define the carbon epoxy composite material. The initial delamination, using a bag insert, was done during the cure processing. After that, the water jet cutting applied to maintain the dimensions following ASTM standards and structural integrity, provoking no defects in the boundaries. DCB (Double-cantilever Beam) and ENF (End-notched Flexure) tests, according ASTM D5528 and ASTM D790-10, run in MTS 250 kN Universal Testing Machine. Scanning Electronic Microscopy demonstrated the damages involved during delamination process. Besides proved that the manual processing provoked many air bubbles, which could increase damage. Modes I and II critical fracture energies (Gıc and $G_{\| c}$ ) are characterized, following the standards, these values are compatible with the literature, therefore the delamination initial energy are identified.

Keywords: Delamination; Composites; Carbon-epoxy.

1 Engenharia de Materiais, estudante, Departamento de Materiais, Centro Universitário da FEl, São Bernardo do Campo, São Paulo, Brasil.

2 Engenharia Mecânica, Dra., Professora, Departamento de Materiais, Centro Universitário da FEI, São Bernardo do Campo, São Paulo, Brasil
\end{abstract}

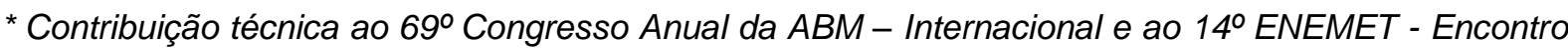
Nacional de Estudantes de Engenharia Metalúrgica, de Materiais e de Minas, 21 a 25 de julho de 2014, São Paulo, SP, Brasil.
} 


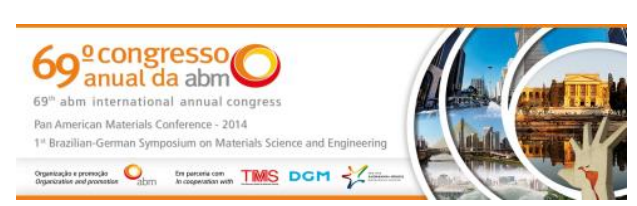

\section{INTRODUÇÃO}

Os materiais compósitos laminados são muito utilizados nas indústrias aeronáutica e aeroespacial. São altamente resistentes e mais leves que as ligas de alumínio usadas atualmente, permitindo a redução da quantidade de material utilizado, no consumo de combustível e na manutenção. Isso acontece em função da resistência e rigidez específicas dos compósitos, que podem ser cinco vezes maiores do que as do alumínio. Porém, estes materiais apresentam, entre outros mecanismos de danos, a delaminação, que é considerado o mecanismo mais preocupante, pois, além de ser subsuperficial, promove a fratura catastrófica [1-3].

Para análise de delaminação de materiais compósitos laminados, o parâmetro mais importante a ser determinado é a energia crítica de fratura (Gc), que é a energia necessária para iniciar a trinca. Para esses ensaios, é feito uma trinca artificial que define como será a propagação. Os testes são executados em função do tipo, ou modos, de abertura da trinca conforme Figura $1[1,4]$.

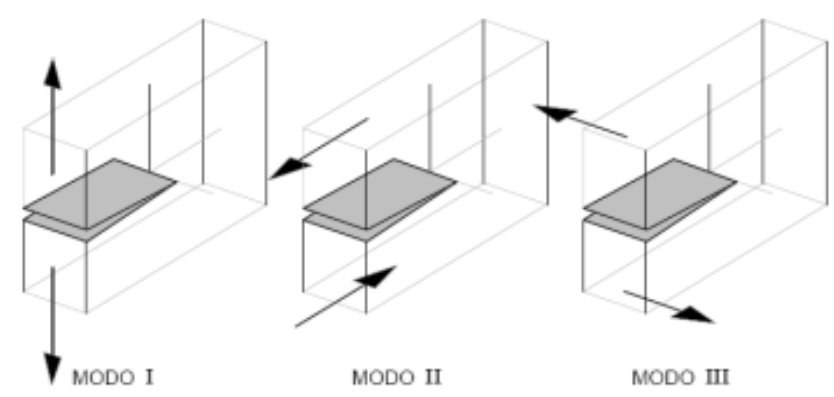

Figura 1. Modos de abertura da trinca.

No presente trabalho, somente serão caracterizados os processos de delaminação em Modo I e II, sendo os testes para essa caracterização, DCB (Double-cantilever Beam) para o Modo I e o ENF (End-notched Flexure) para o Modo II, pois considerase que o Modo III é aproximadamente igual ao Modo II.

\subsection{DCB (Double-cantilever Beam)}

De acordo com Chai [4], o Modo I é o responsável por ampliar o efeito de delaminação. O cálculo do Gıc está representado pela Equação 1, sendo necessário ter o conhecimento da compliância (Equação 2) [4,5].

$$
\begin{gathered}
G_{I C}=\frac{n}{2 b} \frac{k P^{2} a^{n}}{a} \\
C=\frac{\delta}{P}=k \cdot a^{n}
\end{gathered}
$$

Chai [4] realizou ensaios em compósito de epóxi e fibra de carbono multidirecional a fim de estudar o Modo I de delaminação. O corpo de prova utilizado por ele tinha uma trinca inicial de $50 \mathrm{~mm}$, taxa de abertura de $1,3 \mathrm{~mm} / \mathrm{min}$ e lâminas com orientações de $0^{\circ} / 0^{\circ}, 45^{\circ} /-45^{\circ}$ e $90^{\circ} / 90^{\circ}$, variando dimensões e utilizando diferentes resinas epóxis. Os valores médios de Gı encontrados foram de 129,84, 123, 99 e $138 \mathrm{~N} / \mathrm{m}$ para as orientações de $0 \% 0^{\circ}, 45^{\circ} / 45^{\circ}$ e $90^{\circ} / 90^{\circ}$, respectivamente. $\mathrm{O}$ fato de os valores serem inferiores a outros trabalhos, como Salerno [5] e Gill et al. [6], se dá pela falta de agente de compatibilização, como o Silano [4]. Gill et al. [6]

\footnotetext{
* Contribuição técnica ao $69^{\circ}$ Congresso Anual da ABM - Internacional e ao 14ํㅡㄹ ENEMET - Encontro Nacional de Estudantes de Engenharia Metalúrgica, de Materiais e de Minas, 21 a 25 de julho de 2014, São Paulo, SP, Brasil.
} 


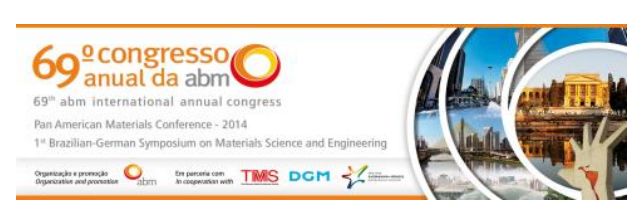

vácuo por uma hora a $120^{\circ} \mathrm{C}$ na estufa Fanem (Figura 3a). O corte a jato d'água promoveu a precisão das dimensões e integridade na lateral do compósito.

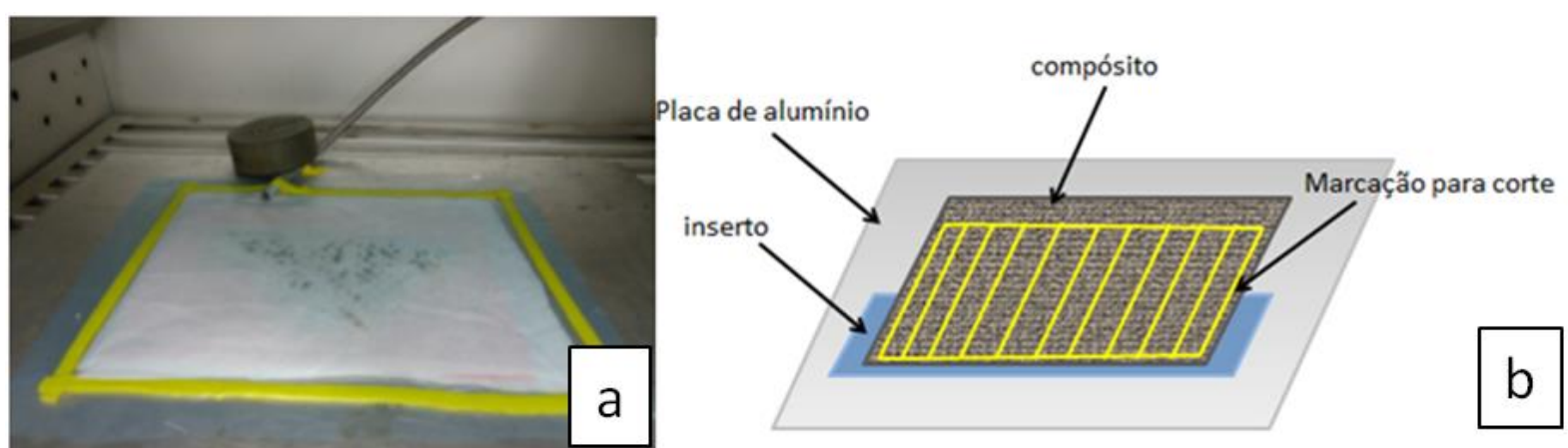

Figura 3. (a) material compósito na estufa. (b) placa de compósito para corte.

Para o cálculo da densidade do compósito, foi cortado $1 \mathrm{~cm}^{2}$ do compósito, medida sua espessura e massa. Para determinar a porcentagem de fibra de carbono (\%F.C.), foi utilizada a massa anterior em recipiente cerâmico; após 1 hora a $500^{\circ} \mathrm{C}$, a resina evaporou e permaneceram somente as fibras.

\subsection{DCB (Double-cantilever Beam)}

O corpo de prova DCB consiste em uma amostra de espessura retangular e uniforme, de um compósito laminado contendo um inserto não adesivo no plano médio da espessura que atua como uma trinca inicial (delaminação). Forças de abertura são aplicadas na amostra através dos blocos que estão colados na extremidade da amostra, Figura 4, causando a separação das camadas. As dimensões dos corpos de prova (Figura 4) foram obtidas pela a norma ASTM D5528 [7] sendo a espessura (h) e o comprimento da trinca inicial (a) calculadas para valores de $a \leq 63 \mathrm{~mm}$ e $\mathrm{h} \geq 3 \mathrm{~mm}$ (considerando $\mathrm{G}_{1 \mathrm{C}}$ e $\mathrm{E}_{11}$, respectivamente, 239 $\mathrm{J} / \mathrm{m}^{2}$ e $\left.20 \mathrm{GPa}[5,10]\right)$. Foram utilizados oito corpos de prova. Foram feitas marcas a cada $1 \mathrm{~mm}$, a fim de medir o deslocamento da trinca. Os blocos foram fixados na amostra (com uma cola epóxi de cura a frio) e na máquina de forma a deixar a amostra alinhada e centralizada. A Figura 5 mostra um corpo de prova pronto para ensaio.

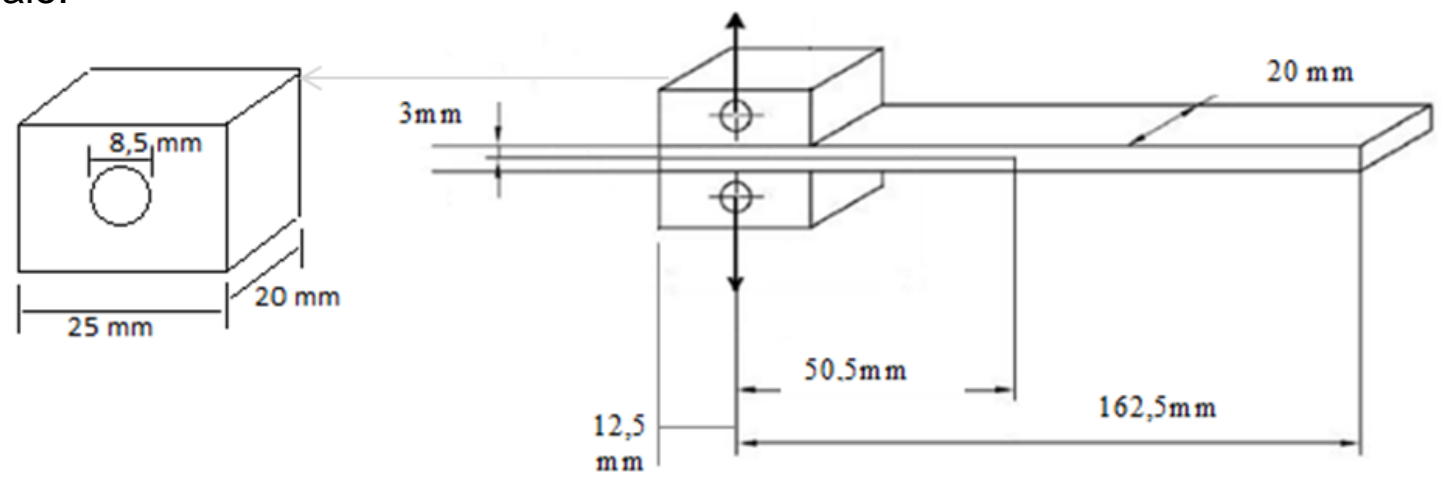

Figura 4. Desenho esquemático do bloco e do corpo de prova usados para o ensaio e DCB, bem como suas dimensões.

Para esse trabalho foi utilizada uma velocidade constante de $2 \mathrm{~mm} / \mathrm{min}$ com o objetivo de garantir que a delaminação ocorra sem a ruptura do compósito, na máquina universal de ensaios MTS com capacidade de $250 \mathrm{kN}$. Para o cálculo do

\footnotetext{
* Contribuição técnica ao $69^{\circ}$ Congresso Anual da ABM - Internacional e ao 14ํㅡㄹ ENEMET - Encontro Nacional de Estudantes de Engenharia Metalúrgica, de Materiais e de Minas, 21 a 25 de julho de 2014, São Paulo, SP, Brasil.
} 


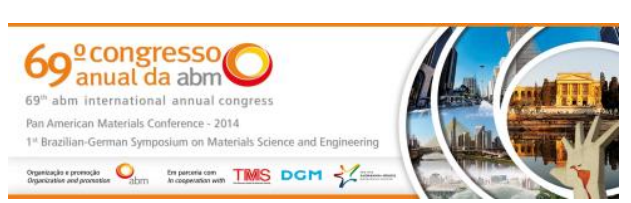

Gıc, deve-se utilizar o ponto de instabilidade do gráfico Força x deslocamento, ou seja, o ponto onde se perde a linearidade dessa relação. Para determinar as constantes $n$ e $k$, foi feito o gráfico do log da compliância (Equação 2) pelo log do comprimento de trinca (imagens capturadas durante o ensaio), sendo $n$ o coeficiente da reta e $0 k$ a constate da equação de reta. Com os valores de força, deslocamento, abertura da trinca e os coeficientes $n$ e $k$, é possível fazer o cálculo do Gıc de acordo com Equação 1.

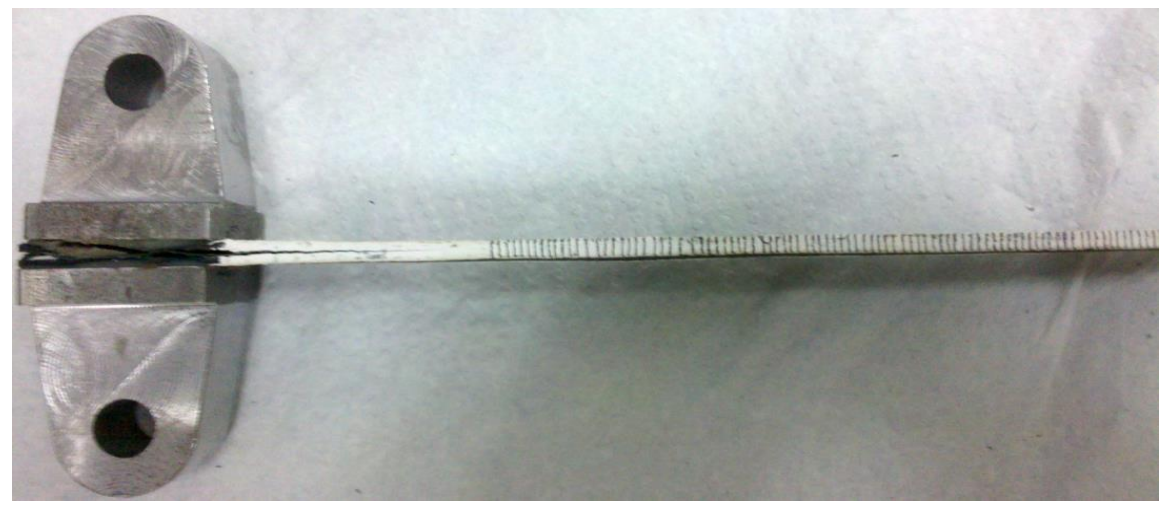

Figura 5. Corpo de prova pronto para ensaio DCB.

\subsection{ENF (End Notch Flexure)}

A norma ASTM D790-10 [11] determina as dimensões das amostras de acordo com a Figura 6. Para o cálculo da trinca inicial a deve-se buscar a estabilidade do teste, sendo essa relação $0,37<(a / l)<1$, onde a e / são, respectivamente o comprimento da trinca e do span. A relação entre span e espessura é de $32: 1$ no caso de compósitos reforçados de alta resistência [11]. Sendo a espessura 3,2 mm, o span foi de aproximadamente $102 \mathrm{~mm}$. Dessa forma, 37,74 $\mathrm{mm}<a<102 \mathrm{~mm}$. Para 0 ensaio, a trinca escolhida foi de $41 \mathrm{~mm}$. Os pinos de apoio e de carga são de diâmetro igual a $15,8 \mathrm{~mm}$. A velocidade utilizada no ensaio foi de $2 \mathrm{~mm} / \mathrm{s}$. Foi utilizado o equipamento Instron de capacidade máxima de $30 \mathrm{kN}$, conectado a um software para obter a curva carga $x$ deflexão, sendo a delaminação obtida por meio de fotos tiradas sincronizadamente ao ensaio. A Figura 7 mostra configuração do ensaio. Para o cálculo do Gıc, foi utilizada a Equação 3 sendo que a força e a abertura de trinca utilizadas devem ser as do ponto de instabilidade da trinca, ou seja, a perda da linearidade entre a força e o deslocamento.

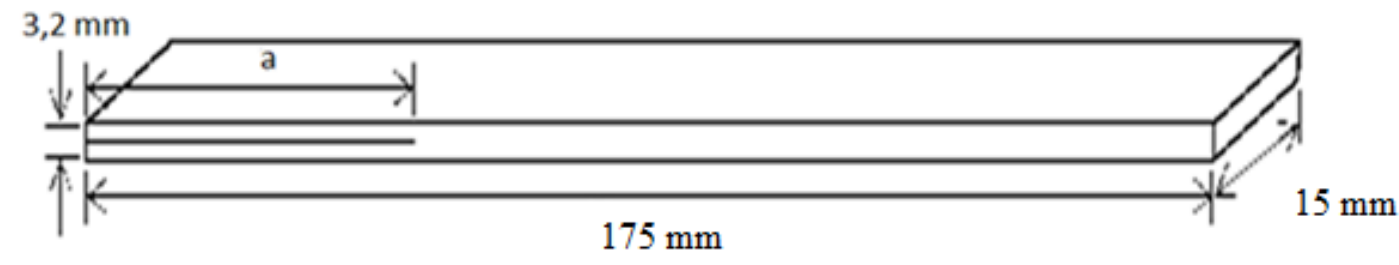

Figura 6. Dimensão da amostra ENF.

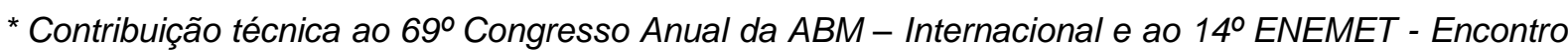
Nacional de Estudantes de Engenharia Metalúrgica, de Materiais e de Minas, 21 a 25 de julho de 2014, São Paulo, SP, Brasil.
} 

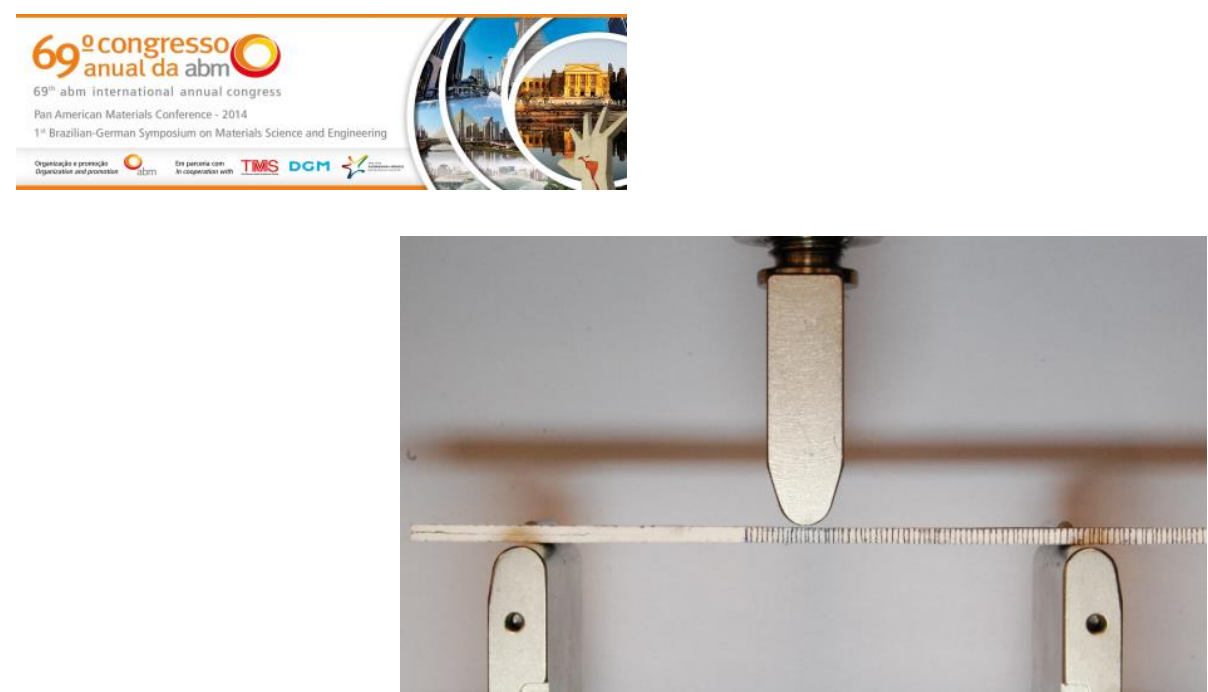

Figura 7. Equipamento preparado para ensaio ENF.

\subsection{Ensaio de Tração}

Foi realizado ensaio de tração para obtenção do módulo elástico $(E)$, coeficiente de Poisson $\left(v_{x y}\right)$, módulo de rigidez $\left(G_{x y}\right)$ e resistência à ruptura $\left(\sigma_{r}\right)$. Foram preparados corpos de prova com as dimensões apresentadas na Figura 8. O ensaio foi realizado na MTS, segundo norma ASTM D3039 [12], com velocidade de $5 \mathrm{~mm} / \mathrm{s}$. Foram posicionados straingages bidirecionais PA-06-125TG-350L no centro do corpo de prova para leitura da deformação longitudinal $(\varepsilon \mid)$ e deformação transversal $(\varepsilon t)$. Foram colados tabs de apoio nas extremidades para a proteção do corpo de prova à pressão da garra (6 MPa). O coeficiente de Poisson e o Módulo de Cisalhamento são obtidos para o plano xy, pois neste o material é isotrópico.

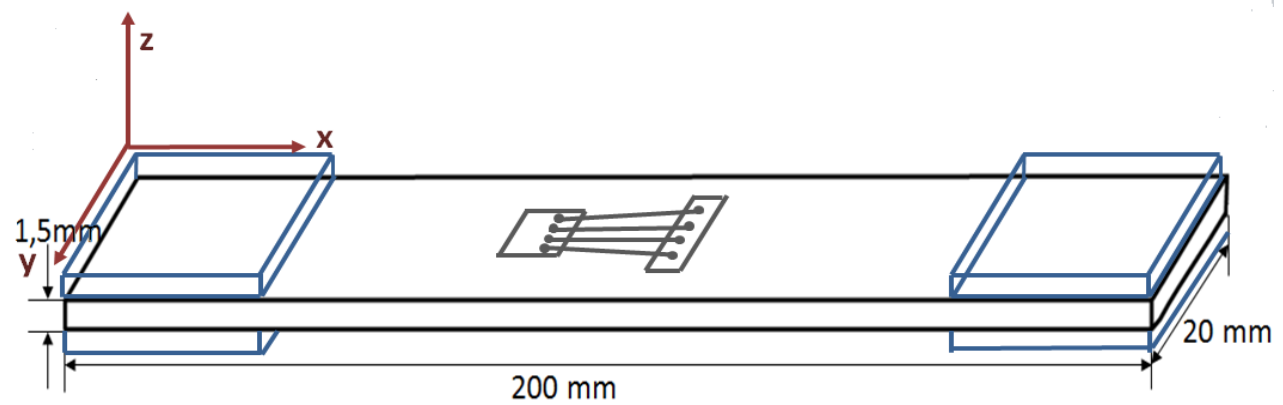

Figura 8. Dimensões dos corpos de prova de tração em preto, tabs de apoio em azul e straingage em cinza.

\section{RESULTADOS E DISCUSSÃO}

A Figura 9 mostra a extremidade do compósito cortado. Pode-se observar que o compósito não apresenta nenhum dano nas extremidades, como delaminações, arrancamento de fibras, entre outros. Outro fato importante é que as dimensões estão precisas, atendendo as especificações das normas ASTM D5528 [7] e ASTM D790-10 [11]. Pode-se notar que o processo de fabricação do inserto utilizando o bag mantém a integridade do compósito, garantindo a trinca inicial.

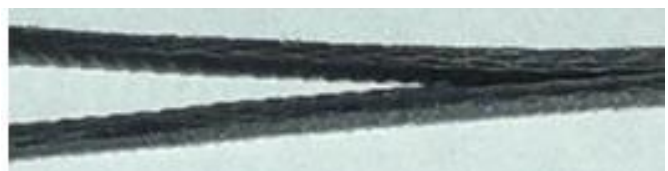

Figura 9. Amostra após corte a jato d'água. Inserto obtido na preparação do compósito.

A densidade e de porcentagem de fibra de carbono foram, respectivamente, $2,2 \mathrm{~g} / \mathrm{cm}^{3}$ e $57,75 \% F . C$. Fato que demonstra que o compósito tem grande

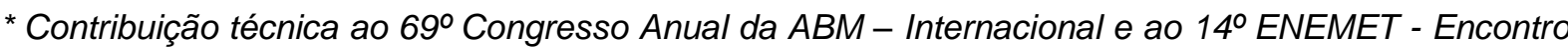
Nacional de Estudantes de Engenharia Metalúrgica, de Materiais e de Minas, 21 a 25 de julho de 2014, São Paulo, SP, Brasil.
} 

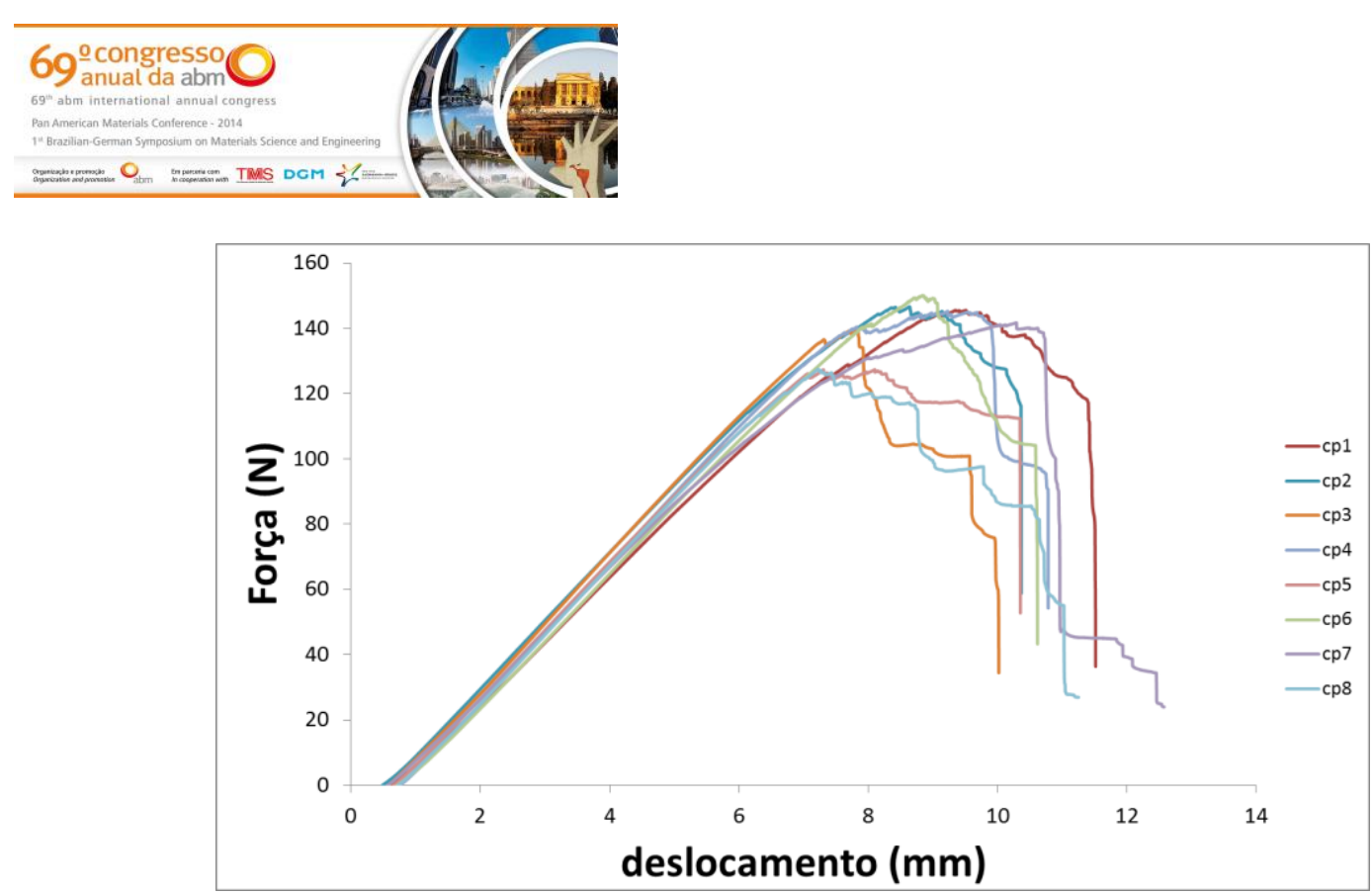

Figura 11. Gráfico Força x deslocamento obtida no ensaio ENF.

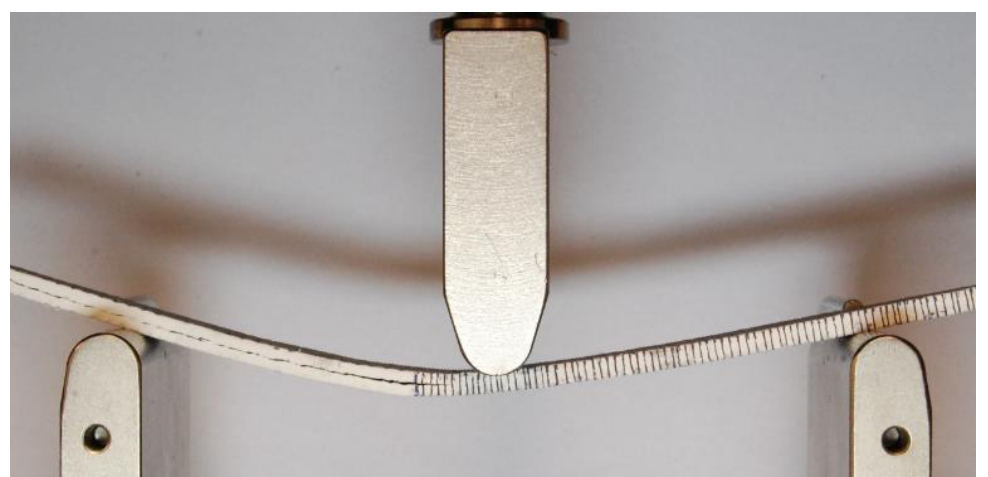

Figura 12. Propagação da trinca $(4 \mathrm{~mm})$ do corpo de prova 1 , apresentando o comportamento de insuficiente delaminação para estudo da propagação da fratura.

A Tabela 2 apresenta os valores da força necessária para iniciar a propagação da trinca, bem como os resultados de energia crítica. Adicionalmente, estão apresentados o valor médio de Gıc e o desvio padrão (Equação 3). O valor da energia crítica necessária para propagar a trinca em Modo II é de $770,72 \mathrm{~J} / \mathrm{m}^{2}$, valor esperado quando comparado com os resultados obtidos por Sela et al [9], $518 \mathrm{~J} / \mathrm{m}^{2}$ para 50\%F.C., enquanto que o compósito estudado neste trabalho possui $57 \%$. Esse fator aumenta a rigidez do compósito o que fez com que o resultado obtido seja maior. Salerno [5] obteve um valor de $837 \pm 235 \mathrm{~J} / \mathrm{m}^{2}$ no compósito unidirecional $0^{\circ} / 90^{\circ}$, que é um resultado superior ao obtido de $770,72 \mathrm{~J} / \mathrm{m}^{2}$, pois no compósito com reforço em tecido há cisalhamento entre as fibras horizontais com as fibras verticais, fazendo, portanto, com que o valor de G॥c possa ser menor em compósitos reforçados com tecido do que nos unidirecionais.

\footnotetext{
* Contribuição técnica ao $69^{\circ}$ Congresso Anual da ABM - Internacional e ao 14ํㅡㄹ ENEMET - Encontro Nacional de Estudantes de Engenharia Metalúrgica, de Materiais e de Minas, 21 a 25 de julho de 2014, São Paulo, SP, Brasil.
} 

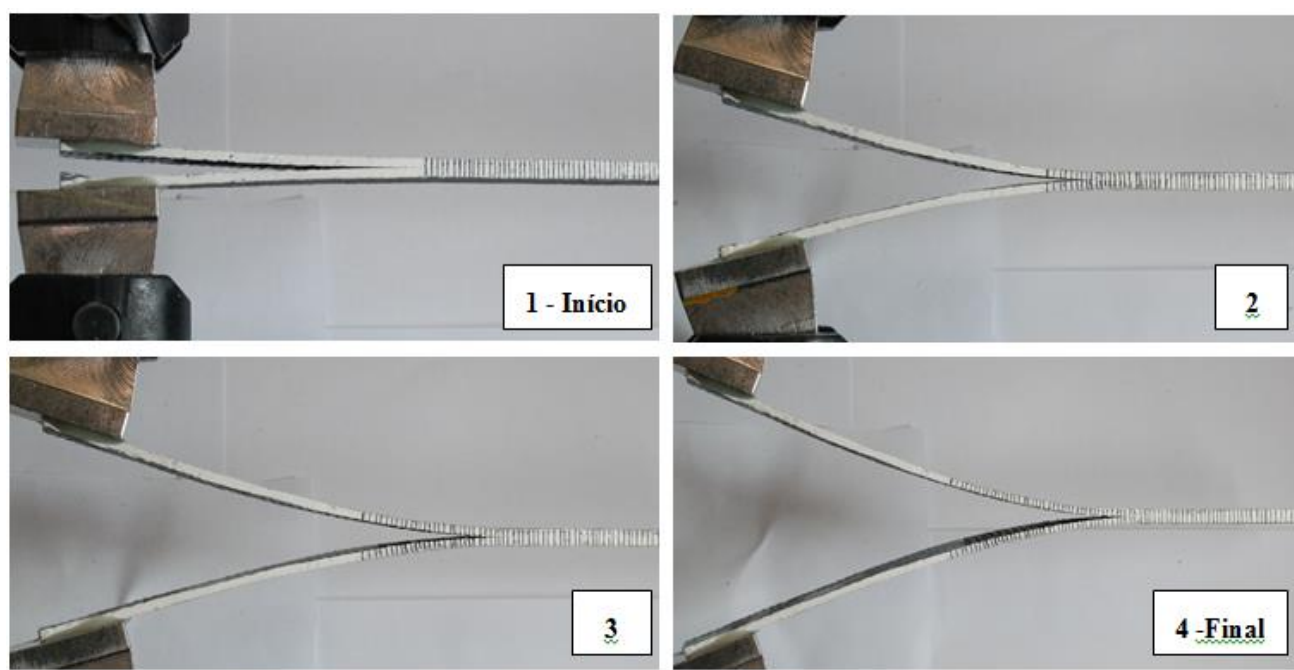

Figura 14. Imagens do corpo de prova 4, enfatizando o comprimento de propagação da trinca.

A Figura 15 mostra os gráficos do logaritmo da Compliância pelo logaritmo do comprimento de trinca para a obtenção dos coeficientes $n$ e $k$ dos oito corpos de prova, que estão apresentados na Tabela 3, bem como os valores de força e Gıc.

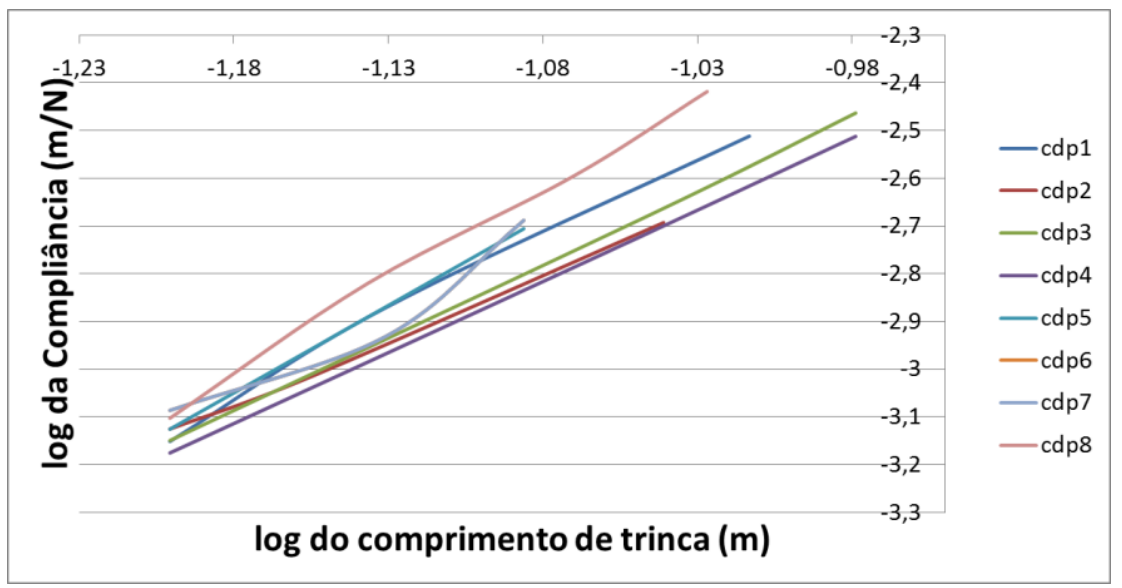

Figura 15. Gráfico log Compliância x log comprimento de trinca.

Tabela 3. valores obtidos de $n$ e $k$, força e $G_{I C}$

\begin{tabular}{ccccc}
$\mathbf{c d p}$ & $\mathbf{n}$ & $\mathbf{k}$ & $\mathbf{P}(\mathbf{N})$ & $\mathbf{G}_{\mathbf{I C}}\left(\mathbf{J} / \mathbf{m}^{\mathbf{2}}\right)$ \\
\hline $\mathbf{1}$ & 2,566 & 0,940 & 27,854 & 468,431 \\
$\mathbf{2}$ & 3,375 & 2,567 & 28,552 & 492,202 \\
$\mathbf{3}$ & 2,732 & 1,407 & 25,290 & 386,160 \\
$\mathbf{4}$ & 3,084 & 3,568 & 33,798 & 689,687 \\
$\mathbf{5}$ & 2,987 & 2,576 & 37,862 & 865,520 \\
$\mathbf{6}$ & 3,021 & 2,012 & 32,071 & 621,005 \\
$\mathbf{7}$ & 3,368 & 2,123 & 28,367 & 485,844 \\
$\mathbf{8}$ & 3,017 & 1,791 & 27,581 & 459,293 \\
\hline média & $\mathbf{3 , 0 1 9}$ & $\mathbf{2 , 1 2 3}$ & $\mathbf{3 0 , 1 7 2}$ & $\mathbf{5 5 8 , 5 1 8}$ \\
desvio padrão \pm & $\mathbf{0 , 3 2 9}$ & $\mathbf{0 , 8 0 4}$ & $\mathbf{4 , 1 0 0}$ & $\mathbf{1 5 7 , 0 7 5}$
\end{tabular}

O valor obtido de Gıc é esperado de acordo com a literatura, em Gill et al [6], para um compósito com 54,4\%F.C., obteve um valor de $525 \mathrm{~J} / \mathrm{m}^{2}$. Valor inferior aos encontrados no ensaio ENF, na Tabela 2. Esse fato é devido a existência do campo de compressão no ENF, enquanto que no DCB se trata de tração direta, o que

* Contribuição técnica ao $69^{\circ}$ Congresso Anual da ABM - Internacional e ao 14ํㅡㄹ ENEMET - Encontro Nacional de Estudantes de Engenharia Metalúrgica, de Materiais e de Minas, 21 a 25 de julho de 2014, São Paulo, SP, Brasil. 


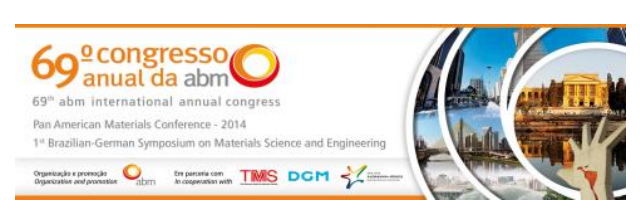

facilita significativamente a abertura da trinca, propagando-a com maior facilidade, fato esse comprovado quando se observa o comprimento de propagação da trinca dos corpos de prova dos ensaios DCB e ENF.

A Figura 16 apresenta o comportamento da energia de abertura de trinca em função do crescimento da trinca. Observa-se dois comportamentos diferentes, sendo um que a energia de abertura de trinca diminui com o crescimento da trinca e o segundo em que o $\mathrm{G}_{\text {I }}$ aumenta com o crescimento da trinca, semelhante ao comportamento apresentado por Gill et al [6]. De acordo com Gill et al [6], a trinca que se propaga na direção transversal à abertura de trinca, tem um aumento de $60 \%$ da força necessária para propagar a trinca na direção da abertura. Isso pode ser observado nas imagens feitas por MEV. A Figura 17a apresenta a imagem de MEV feita para 0 corpo de prova 4, onde de acordo com a Figura 16, observa-se a diminuição da energia de abertura de trinca com o crescimento da trinca. As marcas de fratura na matriz epóxi mostram que o sentido da propagação da trinca é o mesmo do sentido de abertura da trinca. Na Figura 17b, onde mostra o corpo de prova 1, no qual $\mathrm{G}_{\text {I }}$ aumenta conforme ocorre o crescimento da trinca, pode-se observar que em algumas regiões existem marcas de propagação no sentido transversal a abertura de trinca, no qual a propagação transversal pode acontecer por que a energia necessária para atravessar uma fibra na transversal é maior do que acompanhar a direção dessa fibra [6]. Esse comportamento está apresentado na Figura 17b onde a mudança da direção da propagação está exatamente no entrelaçamento das fibras, ou seja, onde há a mudança de direção.

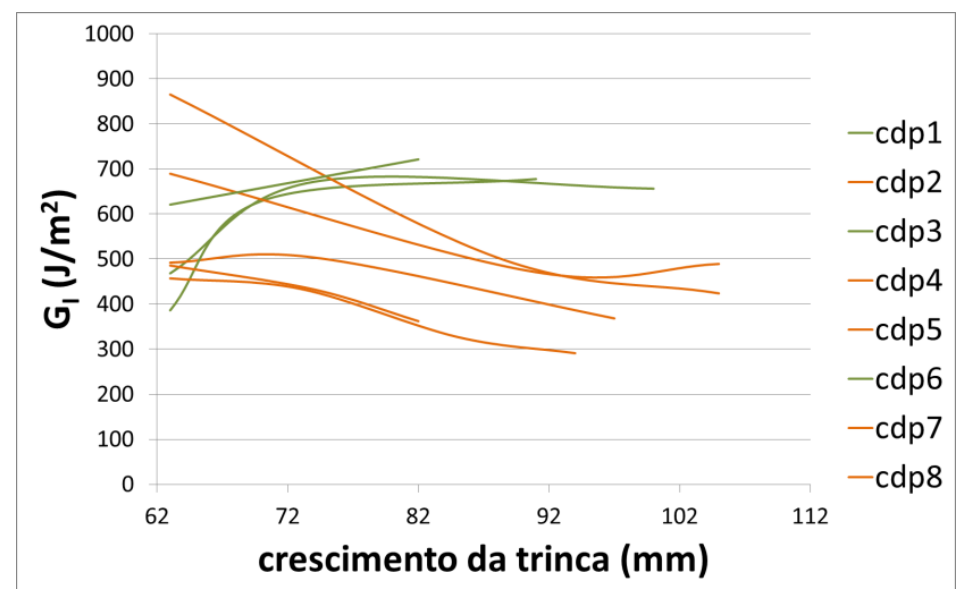

Figura 16. Gráfico $\mathrm{G}_{1} \times$ crescimento da trinca mostrando os dois comportamentos obtidos.
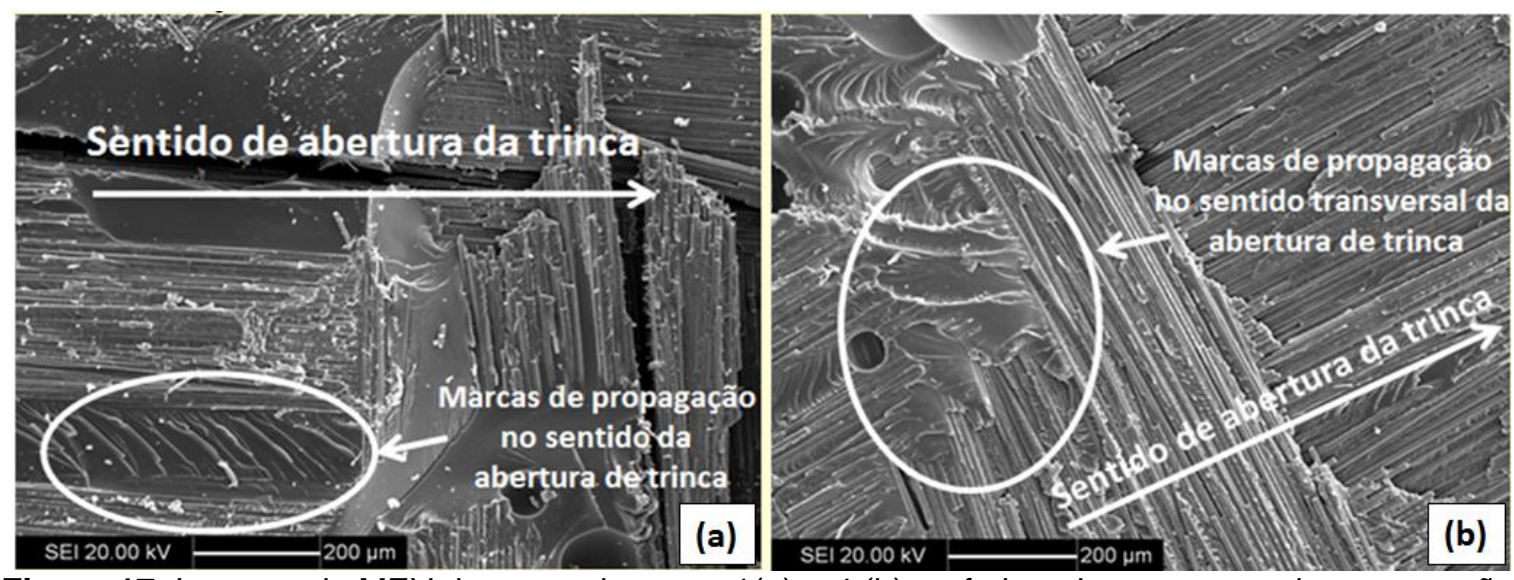

Figura 17. Imagem de MEV do corpo de prova 1 (a) e 4 (b), enfatizando as marcas de propagação.

* Contribuição técnica ao 69 Congresso Anual da ABM - Internacional e ao 14ํㅡㄹ ENEMET - Encontro Nacional de Estudantes de Engenharia Metalúrgica, de Materiais e de Minas, 21 a 25 de julho de 2014, São Paulo, SP, Brasil. 


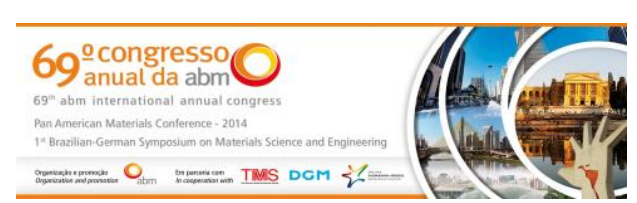

A Figura 18a mostra uma imagem de MEV da região do final do inserto e começo da fratura. É possível observar a planicidade da matriz na região do inserto, enquanto que a região da fratura há arrancamento da fibra, ruptura da matriz, entre outras. Demonstra que a fabricação do inserto atendeu a descrição da norma. Adicionalmente, observa-se existência de bolhas na superfície da fratura do compósito que mostra que a fabricação manual do compósito não foi apropriada, podendo levar a danos. O fato de essas bolhas existirem pode estar relacionado com a pressão insuficiente gerada pela bomba de vácuo, fazendo com que nem todo ar seja eliminado no momento da cura. Na Figura 18b é possível observar que além da fratura da matriz e da delaminação, há fratura das fibras e descolamento da fibra em relação à matriz durante a delaminação.
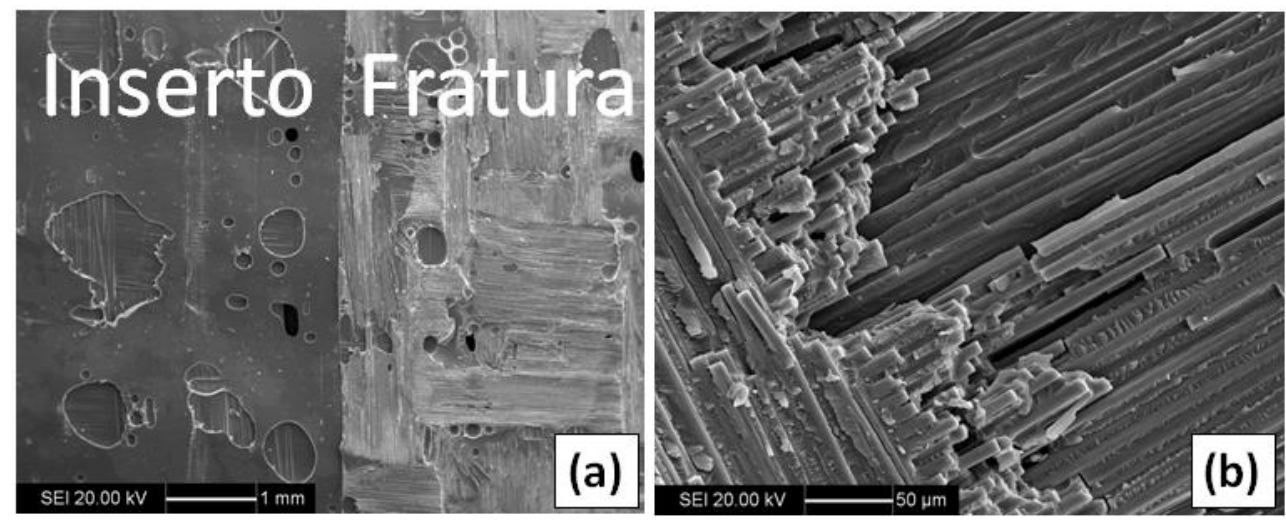

Figura 21. Imagem de MEV enfatizando o final do inserto (a) e fratura das fibras.

\section{CONCLUSÕES}

Pode-se concluir que é viável a fabricação desse compósito, inclusive com o inserto. Porém, a presença de bolhas no compósito mostra que houve falha na preparação, devido a baixa pressão da bomba de vácuo. O corte com o jato d'água foi eficiente para manter a integridade das extremidades mantendo boa qualidade dimensional, sem introduzir defeitos.

$O$ inserto atendeu perfeitamente as exigências da pré-trinca sem danificar ou contaminar o material e evitou a aderência entre as superfícies de cada braço de material compósito.

A densidade do compósito, bem como a porcentagem de fibra, levam o compósito a apresentar um comportamento rígido. Comportamento esse verificado pelas propriedades obtidas em tração e por MEV, onde se observa propagação frágil da trinca.

Os valores de $\mathrm{G}_{\mathrm{IC}}\left(558,528 \mathrm{~J} / \mathrm{m}^{2}\right)$ e $\mathrm{G}_{\text {Ilc }}\left(770,72 \mathrm{~J} / \mathrm{m}^{2}\right)$ obtidos estão dentro do esperado pela literatura. $O$ fato de a energia necessária para iniciar a propagação da trinca em Modo II ser maior do que Modo I é por que, na flexão existe um campo de compressão, enquanto que no Modo I se trata de uma abertura pura por tração. Esse fator faz com que o comprimento de propagação da trinca em Modo II seja significativamente menor do que Modo I, dificultando a caracterização da propagação da fratura. A curva de resistência à delaminação apresenta dois comportamentos de energia de abertura de trinca, sendo que o primeiro diminui enquanto aumenta o comprimento da trinca e o segundo diminui.

\footnotetext{
* Contribuição técnica ao $69^{\circ}$ Congresso Anual da ABM - Internacional e ao 14ํㅡㄹ ENEMET - Encontro Nacional de Estudantes de Engenharia Metalúrgica, de Materiais e de Minas, 21 a 25 de julho de 2014, São Paulo, SP, Brasil.
} 


\section{Agradecimentos}

Ao Centro Universitário da FEI, à Prof ${ }^{a}$ Dra. Gigliola Salerno, ao Prof ${ }^{\circ}$. Msc. Willian Naville pelo auxílio nos ensaios e na utilização da máquina universal de ensaios, à Prof ${ }^{a}$ Dra. Daniella Caluscio pela colaboração e auxílio nas análises por microscopia eletrônica de varredura e aos técnicos do Laboratório de Materiais (LabMat-FEI).

\section{REFERÊNCIAS}

1 Cândido GM, Rezende MC, Donadon MV, Almeida SFM. Fractografia de compósito estrutural aeronáutico submetido à caracterização de tenacidade à fratura interlaminar em Modo I. Polímeros. 2012; 22(1). http://dx.doi.org/10.1590/S010414282012005000019

2 Rezende MC, Costa ML, Botelho EC. Compósitos estruturais. Tecnologia e Prática. São Paulo: Artliber; 2011.

3 Choi NS, Kinloch AJ, Willians JG. Delamination fracture of multidirectional carbonfiber/epoxy composite under Mode I, Mode II and mixed-Mode I/II loading. Journal of Composite Materials. 1999;33(1):73-100.

4 Chai $\mathrm{H}$. The caracterization of Mode I delamination failure in nonwovem, multidirectional laminates. Composites. 1984:5(4):277-290.

5 Salerno G. Damage analysis of composite laminates subject to low velocity impacts. [dissertação de doutorado em Engenharia estrutural]. Milão: Doctoral School in Structural, Politecnico di Milano; 2009.

6 Gill AF, Robinson P, Pinho S. Effect of variation in fibre volume fraction on modes I e II delamination behaviour of $5 \mathrm{HS}$ woven composites. Composites science and technology. 2009;69(14):2368-2375.

7 American Society for Testing and Materials. ASTM D5528: Standard Test Method for Mode I Interlaminar Fracture Toughness of Unidirectional Fiber-Reinforced Polymer Matrix Composites. Standard D. 20017:5528-01.

8 Pankow M, Salvi A, Waas AM, Yen CF, Ghiorse S. Resistence to delamination of 3D woven textile composites evaluated using End Notch Flexure (ENF) tests: experimental results. In: Composites, p. 1463-1476, 2011. Ed: Elsevier Ltda.

9 Sela N, Ishai O. Interlaminar fracture toughness and toughening of laminated composite materials: a review. Composites, 1999; 20.

10 Rossini MB. Caracterização das propriedades elásticas de um material compósito laminado. 2012. f. 73. Dissertação (Graduação em Engenharia de Materiais) - Centro Universitário da FEI, São Bernardo do Campo, 2012.

11 ASTM D790. American Society for Testing and Materials (ASTM). Standard Test Methods for Flexural Properties of Unreinforced and Reinforced Plastics and Electrical Insulating Materials. Standard D, 790-10, 2010.

12 ASTM D3039. American Society for Testing and Materials (ASTM). Standard Test Method for Tensile Properties of Polymer Matrix Composite Materials. Standard D, 3039, 2008.

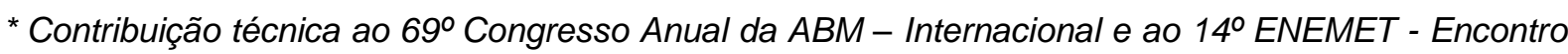
Nacional de Estudantes de Engenharia Metalúrgica, de Materiais e de Minas, 21 a 25 de julho de 2014, São Paulo, SP, Brasil.
} 